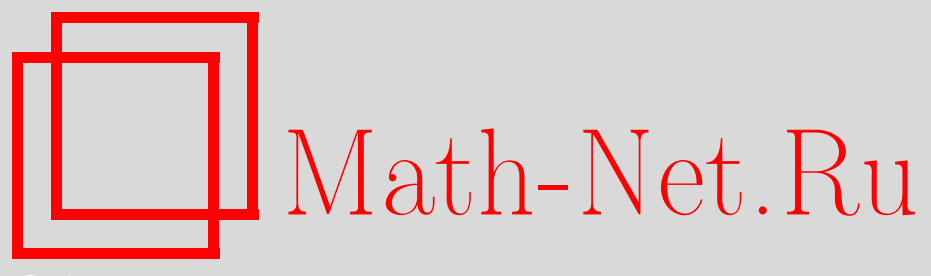

А. А. Бейлинсон, А. С. Вишик, Д. А. Каждан, М. М. Капранов, А. С. Меркурьев, Д. О. Орлов, И. А. Панин, А. А. Суслин, Н. А. Тюрин, Г. Б. Шабат, Владимир Александрович Воеводский (некролог), УМН, 2018, том 73, выпуск 3, 157-168

DOI: https://doi.org/10.4213/rm9821

Использование Общероссийского математического портала Math-Net.Ru подразумевает, что вы прочитали и согласны с пользовательским соглашением http://www . mathnet.ru/rus/agreement

Параметры загрузки:

IP : 18.234 .197 .8

26 апреля 2023 г., 15:09:48

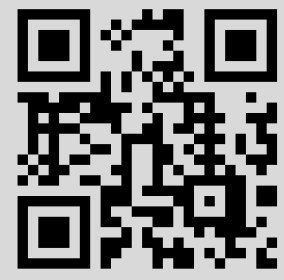




\section{Владимир Александрович Воеводский}

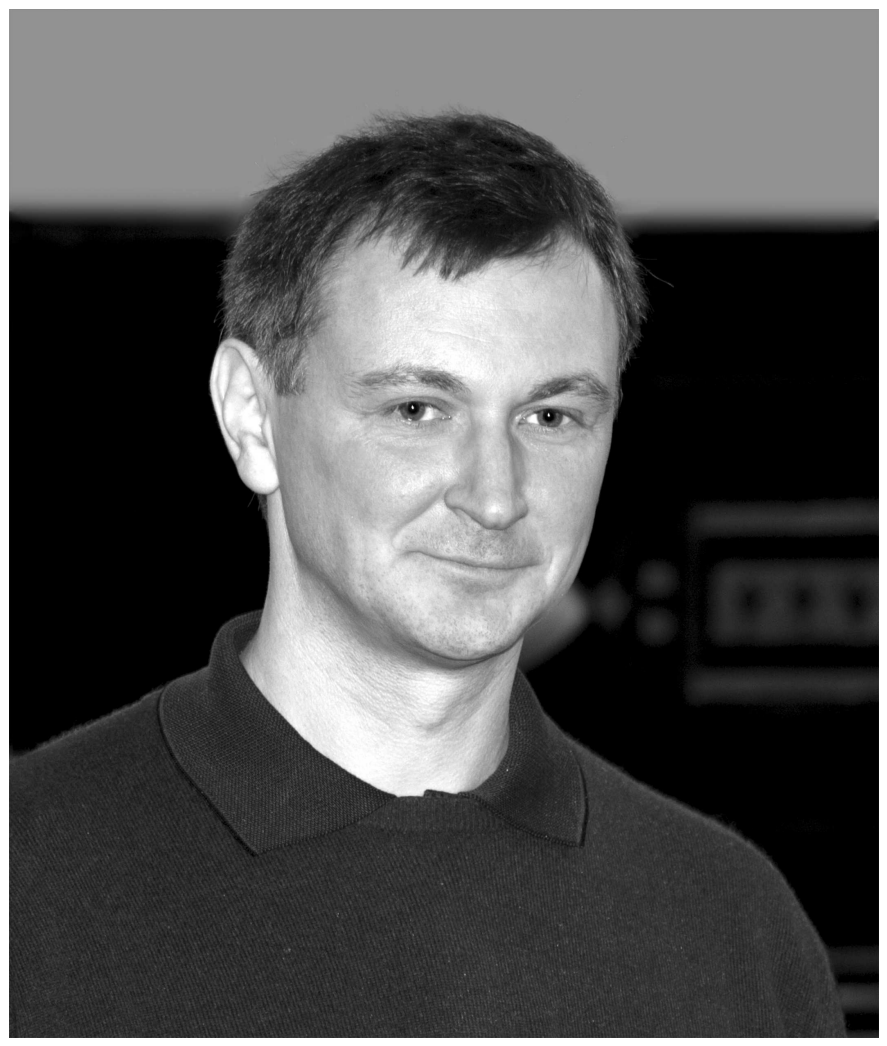

В конце сентября 2017 г. на 52-м году ушёл из жизни выдающийся математик, лауреат премии Филдса, профессор Института перспективных исследований Владимир Александрович Воеводский. Уникальный как личность и как учёный, Владимир Воеводский оставил богатое наследство в математике, истинную величину которого пока ещё трудно оценить. Обладая редким научным даром предвидения и обобщения, он видел значительно дальше других, и его всегда отличали широта и свобода взгляда на предмет и смелость в выборе путей исследования. В результате его идеи принципиально изменили несколько областей математики, а работы привели к созданию новых направлений в математике и компьютерных науках.

Открытый в общении и предельный в честности, полный замечательных замыслов, которыми щедро делился с друзьями и коллегами, Владимир всегда был яркой индивидуальностью в науке и в жизни, центром притяжения, заражающим энергией и увлекающим к новым горизонтам.

DOI: https://doi.org/10.4213/rm9821 
Владимир Воеводский родился в научной семье с богатыми традициями; он рано проявил склонность к приобретению знаний, ещё в детстве серьезно увлекаясь химией. Как сам он впоследствии рассказывал, сначала интерес к химии необходимо привел его к атомной физике, без которой невозможно было разобраться в химии, а затем интерес к физике привел его в математику, без которой, в свою очередь, невозможно было разобраться в физике. В 1983 г. Владимир поступает на механикоматематический факультет МГУ, однако не все обязательные курсы вызывают у него интерес, что приводит к тому, что Владимир дважды был отчислен с факультета; второй раз, и уже окончательно, с четвёртого курса. Многим вокруг уже казалось, что математика - это не совсем правильный выбор. Однако сам Владимир так не считал, а несколько неформальных встреч с И. Р. Шафаревичем дали ему огромный дополнительный импульс. Игорь Ростиславович не только разглядел в не очень успевающем студенте незаурядный талант, но и, правильно оценив, точно направил его к другому, близкому ему по духу и восприятию математики человеку - Александру Гротендику. С первой встречи с И. Р. Шафаревичем Владимир пришёл с книгой (неформальными записями) А. Гротендика, ради изучения которой был за три месяца выучен французский язык.

Именно с этого началась серьезная научная работа, в жертву которой было принесено формальное обучение (Владимир является, наверное, единственным филдсовским лауреатом, так и не получившим формального "высшего образования"). Под руководством Г.Б. Шабата и в сотрудничестве с ним Владимир начинает работать над программой А. Гротендика, связывающей “детские рисунки” (т. е. графы на римановой поверхности) с алгебраическими кривыми, определёнными над полями алгебраических чисел. Совместно с Г. Б. Шабатом были написаны две работы [1], [2], из которых вторая, "Рисуя кривые над числовыми полями", была опубликована в сборнике, посвящённом 60-летию Александра Гротендика. Юбиляр лично поблагодарил авторов статьи, что произвело на младшего соавтора (Владимира) огромное впечатление, заставило его поверить в свои собственные возможности и подтвердило выбор "гротендиковского стиля" не только в науке, но и в жизни. И этот “гротендиковский уровень" оказался Владимиру по плечу: ему было суждено развить и претворить в жизнь некоторые фундаментальные идеи Александра Гротендика, до того казавшиеся слишком смелыми.

Занимаясь алгебраической геометрией, Владимир принимает активное участие в семинаре И. Р. Шафаревича в Математическом институте им. В. А. Стеклова и в семинаре Ю.И. Манина на механико-математическом факультете МГУ. Совместно с учеником последнего, Михаилом Капрановым, он изучает $n$-категории и их связь с теорией гомотопий. Построение теории $n$-категорий являлось важной открытой проблемой, над которой параллельно с ними работали многие математики. Здесь Воеводскому и Капранову удаётся получить много важных и интересных результатов, которые были опубликованы в серии совместных работ [4]-[7], [10], [11].

Одновременно, с конца 80-х годов Владимир Воеводский начинает работать над построением категории мотивов. Идея мотива была выдвинута Александром Гротендиком в 60-е годы. Гротендик предположил, что должен существовать алгебро-геометрический вариант гомотопической категории, который позволит напрямую применить весь аппарат топологии к решению алгебраических проблем. Местом наиболее тесного соприкосновения алгебраической геометрии и топологии долгое время была $K$-теория. Построенный Даниелем Квилленом алгебраический вариант этой теории давал обширный материал для топологических ассоциаций. С другой стороны, созданная классиками теория пересечений алгебраических циклов сделала из групп Чжоу полноценную теорию когомологий, которая была расширена до высших групп Чжоу Спенсером Блохом. Эти группы Блоха были предложены в качестве алгебро-геометрического аналога сингулярных когомологий в топологии. Фундаментальные гипо- 
тезы, выдвинутые Александром Бейлинсоном, Стивеном Лихтенбаумом и Кристофером Суле, обозначили важные ориентиры для дальнейшего развития данной области. В частности, предполагалось существование алгебраического варианта спектральной последовательности Атьи-Хирцебруха, сходящейся от высших групп Чжоу к алгебраической $K$-теории. Кроме того, сами высшие группы Чжоу интерпретировались как когомологии некоторых гипотетических комплексов пучков в топологии Зариского. Эти комплексы должны были представлять специальные объекты - мотивы Тэйта. Наиболее важная часть гипотез касалась взаимодействия топологии Зариского с этальной топологией. Гипотеза Бейлинсона-Лихтенбаума описывала соответствующие комплексы с конечными коэффициентами через пучки этальных когомологий. Отсюда можно было вывести много важных и интригующих следствий.

Таким образом, существовал вполне чёткий план того, что хотелось бы получить, но отсутствовал аппарат, который был бы достаточно гибок для такого построения. И именно такой аппарат был создан Владимиром Воеводским. В 1990 г. он поступает в аспирантуру математического факультета Гарвардского университета (США). В те годы советские математики начали свободно ездить на Запад, восстановление нормальных контактов всячески поддерживалось, - и если была возможность помочь яркому студенту с поступлением в аспирантуру, то это делалось с радостью. Давид Каждан оценил талант необычного аспиранта и стал его научным руководителем. Его поддержка, значение которой трудно переоценить, позволила Владимиру полностью сосредоточиться именно на интересовавших его вопросах и исследованиях. За два года обучения в аспирантуре идеи, зародившиеся ещё в Москве, выкристаллизовываются в построенную Владимиром мотивную категорию.

Эти новые идеи исходили из того, что искомая категория мотивов над данной базой должна получаться локализацией производной категории пучков абелевых групп над ней по интервалу - пучку, представленному в категории соответствий аффинной прямой. Такая локализация эквивалентна стягиванию отрезка в точку в гомотопической топологии. Необходимость работы с пучками в алгебраической геометрии связана с тем, что алгебраические многообразия являются очень жёсткими объектами, в частности, их нельзя склеивать подобно топологическим пространствам; иными словами, в этой категории отсутствуют прямые пределы. Данная проблема устраняется погружением многообразий в категорию пучков в виде представимых пучков. В такой категории склеивать можно всё, и это было понято и описано ещё Гротендиком, который создал богатую теорию пучков в наиболее широком контексте. Пучки в этом смысле зависят от выбора топологии Гротендика на данной категории; в случае алгебраических многообразий это означает, что имеется некоторый выбор понятия "непрерывность". Стандартная непрерывность соответствует топологии Зариского, но имеются и нестандартные варианты. Гротендик ввёл такие топологии, как этальная и плоская, нашедшие в дальнейшем множество замечательных применений.

В своей диссертации "Гомологии схем и ковариантные мотивы" [12] Владимир Воеводский определяет новые топологии, такие как $h$-топология и $q f h$-топология, а также вводит и описывает с их помощью мотивную категорию (она в дальнейшем будет именоваться этальной мотивной категорией). Данные топологии содержат в качестве покрытий раздутия и разбиения на компоненты, т. е. все инструменты, необходимые для разрешения особенностей. Поэтому они позволяют эффективно работать с негладкими объектами. Подход Владимира Воеводского отличался тем, что его мотивная категория являлась триангулированной, в то время как вышеупомянутые гипотезы относились к абелевой категории мотивов. Для построения возможного абелева варианта оказывается необходимым дополнительное данное, $t$-структура. Построенная Владимиром категория была целочисленной. Как он показал, $t$-структура может существовать лишь в случае рациональных коэффициентов. (Её существование это одна из основных гипотез в данной области.) Дальнейшая практика подтверди- 
ла, что триангулированная версия обладает необходимой гибкостью и достаточной универсальностью для большинства задач в случае конечных коэффициентов.

Успешно окончив аспирантуру в 1992-м, Владимир проводит следующий академический 1992-1993-й год в Институте перспективных исследований в Принстоне. Здесь он продолжает изучение мотивов; в частности, приходит понимание, что созданная категория - это этальная версия и для построения основной категории мотивов Зариского нужны дополнительные соображения. В работе "Триангулированные категории мотивов над полем" [23] Владимир Воеводский решает эту проблему. Ключевые новые инструменты в его конструкциях - топология Нисневича и пучки с трансферами. Такая топология, заключённая между этальной топологией и топологией Зариского (где упорядочение топологий понимается как упорядочение непрерывности в смысле одной и другой топологий), удивительным образом наследует важные черты обеих. Примерно в это же время альтернативные конструкции мотивной категории были предложены Марком Левином и Масаки Ханамурой. Однако конструкция В. Воеводского получила более широкое распространение благодаря более гибкому инструментарию, что не замедлило отлиться в ярких приложениях. Статья Владимира Воеводского опирается на несколько его совместных работ с Андреем Суслиным и Эриком Фридландером, с которыми он начинает активно сотрудничать в это время. В частности, при определении пучков с трансферами Владимир опирается на категорию конечных соответствий, введённую А. Суслиным в середине 80-х. Совместная работа с Андреем Суслиным началась ещё раньше, со статьи "Сингулярные гомологии абстрактных алгебраических многообразий” [15], в которой доказывается, что гомологии Суслина комплексного многообразия с конечными коэффициентами совпадают с топологическими гомологиями. Как раз здесь полезной оказалась $h$-топология, заместившая подход, связанный с техникой общего положения (в алгебраической геометрии, в отличие от топологии, утверждения о том, что цикл можно привести в общее положение заменой на эквивалентный, представляют, как правило, существенную трудность). Все эти работы: "Относительные циклы и пучки Чжоу" (совм. с А. Суслиным) [20], "Когомологическая теория предпучков с трансферами" [21], "Бивариантные когомологии циклов" (совм. с Э. Фридландером) [22] выходят в одном сборнике "Циклы, трансферы и мотивные теории гомологий" [24] вместе с уже упомянутой выше работой [23].

В 1993-м Владимир получает трёхгодичную постдокторскую позицию в Гарварде. Такая престижная позиция без преподавания позволит ему на три года сконцентрироваться на одной математике. Эта возможность была реализована в превосходной мере: за эти три года он получит одни из самых блестящих своих результатов.

Продолжая работать над конструкциями из сборника с А. Суслиным и Э. Фридландером, Владимир Воеводский начинает активно заниматься гипотезой Блоха-Като. Этому способствуют его тесные контакты с Андреем Суслиным, который к тому времени имел огромный опыт работы над данной темой и в 1994 г. очень кстати проводил некоторое время в Гарварде в качестве приглашённого профессора. Гипотеза Блоха-Като утверждает, что этальные когомологии поля (т. е. групповые когомологии его абсолютной группы Галуа) с $\mathbb{Z} / p$-коэффициентами совпадают с $K$-теорией Милнора данного поля по модулю $p$. Напомним, что $K$-теория Милнора - это фундаментальный инвариант поля, определяемый удивительно простым образом как квадратичная алгебра над целыми числами с образующими из мультипликативной группы поля и соотношениями вида $a \cdot(1-a)=0$. На мотивном языке $K$-группы Милнора $K_{i}, i \geqslant 0$, могут быть определены как диагональная часть мотивных когомологий точки. Отметим, что в алгебраической геометрии аналоги сфер нумеруются двумя числами (а не одним, как в топологии) в связи с тем, что имеются два разных аналога окружности: обычная симплициальная, как в топологии, и прямая без точки. Умножая в подходящем смысле эти окружности в разных пропорциях, мы и получаем 
такие "алгебраизированные" сферы. Мотивные когомологии - это группы отображений в соответствующей мотивной категории из мотива многообразия в редуцированные мотивы этих сфер, называемые мотивами Тэйта. В результате мотивные когомологии нумеруются двумя номерами: "квадратным" и "круглым". Тогда группа с "диагональным" номером $(i)[i]$ - это в точности $i$-я $K$-теория Милнора. В то же время $i$-е этальные когомологии с конечными коэффициентами отождествляются с этальными мотивными когомологиями с тем же номером $(i)[i]$. Поэтому, сформулированная на этом языке, гипотеза Блоха-Като утверждает, что обычные мотивные когомологии точки с диагональными номерами совпадают со своим этальным аналогом. Таким образом, гипотеза Блоха-Като оказывается частным случаем гипотезы Бейлинсона-Лихтенбаума, которая утверждает то же самое и про все "поддиагональные" номера $(i)[j], i>j$, и про все многообразия, а не только про точку.

В совместной с А. Суслиным работе "Гипотеза Блоха-Като и мотивные когомологии с конечными коэффициентами” [25] Владимир Воеводский выводит всю гипотезу Бейлинсона-Лихтенбаума из данного частного и формулируемого в элементарных терминах случая. Важным инструментом здесь является новая $c d h$-топология, вводимая в этой работе. Объединяя в себе черты топологии Нисневича и $h$-топологии, она даёт возможность работать с мотивами Зариского негладких многообразий. На тот момент гипотеза Блоха-Като была доказана только в малых степенях: случай степени два для всех простых был доказан А. Меркурьевым и А. Суслиным; теми же авторами был сделан случай степени три, отвечающий простому числу 2. Последний результат был независимо получен М. Ростом, который, кроме этого, предложил доказательство случая степени четыре и $p=2$ (не опубликовано). Во всех этих работах, начиная с основополагающей статьи А. Меркурьева про случай $n=2, p=2$, ключевым инструментом было использование так называемых норменных многообразий, а также $K$-теории Квиллена. Идея доказательства состояла в том, чтобы сделать $n$-ю $K$-теорию Милнора $p$-делимой, искусственно убивая все её аддитивные образующие, так называемые "чистые символы". Достигалось это переходом к расширению базового поля, получаемому как композит полей функций норменных многообразий, отвечающих всем чистым символам (поле функций норменного многообразия является универсальным расширением, убивающим данный символ по модулю $p$ ). Далее проверялось, что у такого поля обращаются в нуль не только $K$-теория Милнора по модулю $p$, но и этальные когомологии $c \mathbb{Z} / p$-коэффициентами, откуда следовало, что гипотеза выполняется в этом случае. Наконец, с помощью спуска, гипотеза проверялась тем самым и над базовым полем. Основную трудность такого метода представляла как раз проверка корректности перехода от поля функций норменного многообразия к базовому полю, - здесь и возникала необходимость в $K$-теории Квиллена.

Владимир Воеводский перелагает этот подход на более гибкий мотивный язык. При этом появляются новые объекты; в частности, одну из ключевых ролей отныне будет играть так называемая симплициальная схема Чеха многообразия. Являясь аналогом стягиваемого пространства $E G$ в топологии, она также будет стягиваема, но только если у многообразия имеется рациональная точка. Если же её нет, то это бесконечномерный объект, "измеряющий" отклонения от существования рациональной точкой. Для норменного многообразия этот объект несёт такую же информацию, как и сам символ. Вместе с тем Воеводский замечает, что $K$-теория Квиллена может помочь только в малых степенях, а в общем случае нужно использовать её высшие аналоги: $K$-теории Дж. Моравы $K(n)$. Теории Моравы зависят от простого числа $p$, причем первая из них является прямым слагаемым $p$-локализации обычной $K$-теории. При $p=2$ ситуация была более ясной, так как в этом случае были известны норменные многообразия для любого $n$ - ими являются квадрики Пфистера. Эти "наилучшие из существующих" квадрики находятся во взаимно однозначном соответ- 
ствии с чистыми символами. Маркус Рост показал, что классический мотив Чжоу такой квадрики распадается в прямую сумму скрученных на мотивы Тэйта копий гораздо более маленького мотива, который над замыканием распадается в сумму всего двух мотивов Тэйта. Как установил Владимир Воеводский, мотив Роста в триангулированной категории мотивов может быть представлен как расширение двух копий мотива симплициальной схемы Чеха нашей квадрики.

Комбинируя этот факт с использованием дифференциалов в спектральной последовательности, сходящейся от мотивных когомологий симплициальной схемы к её $n$-й $K$-теории Моравы, Владимир уже в 1995 г. в работе "Гипотеза Блоха-Като с $\mathbb{Z} / 2$ коэффициентами и алгебраическая $K$-теория Моравы" ${ }^{1}$ вывел случай $p=2$ гипотезы (точнее, первоначальный её вариант - гипотезу Милнора) из гипотетического существования этих высших $K$-теорий.

Некоторое время спустя Владимир Воеводский приходит к заключению о том, что в указанной схеме доказательства нужны, собственно, не сами $K$-теории Моравы, а только первые дифференциалы соответствующих спектральных последовательностей. Данные дифференциалы представляются специальными операциями Милнора в мотивных когомологиях с конечными коэффициентами, они выражаются через квадраты Стинрода и действуют как дифференциалы (квадрат равен нулю). Более того, если многообразие обладает подходящими характеристическими числами, то указанные дифференциалы задают точную последовательность в мотивных когомологиях соответствующей редуцированной схемы Чеха. Оказывается, что квадрики Пфистера имеют как раз нужные характеристики, что позволяет провести доказательство (а на самом деле и вычислить кольцо когомологий полностью).

В работе "Операции приведённых степеней в мотивных когомологиях" [29] Владимир Воеводский строит мотивную алгебру Стинрода и операции Милнора вместе с ней. В результате он получает безусловное доказательство гипотезы Милнора, впоследствии опубликованное в работе "Мотивные когомологии с $\mathbb{Z} / 2$-коэффициентами" [30]. Этот замечательный результат сразу приковывает всеобщее внимание к мотивной деятельности Воеводского. В скором времени, применяя ту же технику, удаётся доказать и вторую гипотезу Милнора (о квадратичных формах), которая утверждает, что $K$-теория Милнора по модулю 2 изоморфна также и градуированному кольцу Витта квадратичных форм [33] (кольцо Витта составлено из классов анизотропных квадратичных форм над данным полем). Данный изоморфизм влечёт много сильных результатов про квадратичные формы. В доказательстве гипотезы Милнора используются когомологические операции, а с ними и алгебро-геометрический аналог нестабильной гомотопической категории. Такая категория была построена в совместной статье Владимира Воеводского с Фабьеном Морелем “ $\mathbf{A}^{1}$-гомотопическая теория схем" [17]. В этой фундаментальной статье авторы вводят все необходимые инструменты для работы с алгебраическими многообразиями гомотопическими методами. Следуя подходу Квиллена, нужная категория строится как гомотопическая категория некоторой замкнутой модельной категории, в которой объектами являются симплициальные пучки в топологии Нисневича, а локализация отвечает стягиванию в точку аффинной прямой. Построенная в [17] категория является аналогом нестабильной гомотопической категории в классической топологии. Фундаментальная работа, проделанная в [17], позволила Владимиру Воеводскому построить стабильную мотивную гомотопическую категорию $S H(S)$ для произвольной нётеровой схемы $S$ [16]. Данную категорию часто называют стабильной мотивной гомотопической категорией Воеводского (или Воеводского-Мореля). Владимир Воеводский представляет эту новую гомотопическую мотивную технику в своём пленарном докладе на Международном конгрессе математиков в Берлине в 1998 г. [16], сопровождая её новыми блестящими результатами, которые не могли бы быть получены без данной техники. Выбранный

\footnotetext{
1Это первый, опубликованный в виде препринта, вариант статьи [30].
} 
В. Воеводским подход позволяет систематически определять и описывать обобщённые теории когомологий в алгебраическом контексте и работать с ними ровно так же, как и в классической топологии ${ }^{2}$. В частности, таким образом получаются альтернативные описания мотивных когомологий и алгебраической $K$-теории, а также строится новая теория когомологий (теория алгебраических кобордизмов), которая применяется в доказательстве гипотезы Милнора. Созданный язык, методы, глубокие идеи, заложенные в основания, были немедленно восприняты частью математического сообщества, что привело к решению целого ряда классических проблем, не имевших ничего общего на первый взгляд с языком мотивных пространств.

В то же самое время Владимир Воеводский продолжает работать над общим случаем гипотезы Блоха-Като для случая произвольного $p$. Основная трудность общего случая в сравнении со случаем $p=2$ кроется в отсутствии "хороших" норменных многообразий. Эта проблема была решена Маркусом Ростом, построившим такие многообразия со всеми нужными свойствами. После этого можно было применить общую схему доказательства, предложенную Владимиром Воеводским ещё в 1995 г., но по ходу дела возникли и другие дополнительные сложности. Аналог мотива Роста, так называемый обобщённый мотив Роста, теперь состоял не из двух мотивов симплициальных схем Чеха, а из $p$ таких мотивов. Чтобы работать эффективно с такими объектами, нужна была теория мотивов над симплициальной базой; а чтобы показать, что этот мотив является прямым слагаемым в мотиве норменного многообразия, требовалось не только построить конкретные операции Милнора, но и полностью расклассифицировать бистабильные операции, а также некоторые нестабильные.

Это требовало разработки новых обширных сюжетов. Появляется серия статей: "Мотивы над симплициальными схемами" [37], "Симплициальные раддитивные функторы" [38], "Мотивные пространства Эйленберга-Маклейна" [39]. Здесь Владимир Воеводский строит пространства Эйленберга-Маклейна, представляющие мотивные когомологии в гомотопической категории Мореля-Воеводского, и вычисляет их когомологии, что даёт необходимое описание когомологических операций. Отсюда выводится, что обобщённый мотив Роста может быть построен как $(p-1)$-я симметрическая степень бинарного мотива, полученного расширением двух копий мотива симплициальной схемы Чеха многообразия Роста с помощью явного морфизма, описанного в терминах операций Милнора. Более того, такой мотив действительно оказывается прямым слагаемым в мотиве нашего норменного многообразия. Такое описание даёт сильные ограничения на когомологии симплициальной схемы Чеха и, в комбинации с точностью операций Милнора, завершает доказательство гипотезы Блоха-Като.

Доказательство это было опубликовано в работе "О мотивных когомологиях c $\mathbb{Z} / l$ коэффициентами" [42]. Отсюда вытекает такое сильное следствие, как гипотеза Бейлинсона-Лихтенбаума. Эти результаты кардинально изменили ландшафт самой $K$-теории и прилегающих к ней областей.

В 2001 г. Владимир Воеводский получает постоянную позицию профессора Института перспективных исследований в Принстоне, после трёхлетнего пребывания в качестве приглашенного исследователя. В 2002-м на Международном конгрессе математиков в Пекине ему присуждается медаль Филдса за огромный вклад в создание новых теорий когомологий алгебраических многообразий. В своих работах Владимир Воеводский построил целый новый "мотивный мир", тенью которого является мир топологический. Он снабдил математика, входящего в этот новый мир, набором необходимых инструментов, а доказательство гипотезы Блоха-Като подтвердило силу и практическую пользу новых методов. Появилась целая область математики с массой новых задач и перспективных направлений. Некоторые из них Владимир Воеводский намечает сам в статье "Открытые вопросы мотивной стабильной

2 Дж. Ф. Жардин полностью прописал все детали конструкции В. Воеводского категории $S H(S)$ в статье: J.F. Jardine, "Motivic symmetric spectra", Doc. Math., 5 (2000), 445-552. 
гомотопической теории" [26] и в неопубликованных заметках 2001 г. "Notes on framed correspondences" ${ }^{3}$. В этих неопубликованных заметках Владимир Воеводский закладывает основы совершенно нового подхода к построению стабильной мотивной гомотопической категории. Вот что он пишет во введении: "Я надеюсь, что конструкции, описанные в этих заметках, приведут к новой модели стабильной гомотопической теории, которая будет более дружественной для вычислений. Конечно, можно ожидать, что будет нетривиальной задачей показать, что новая и старая модели согласованы". В сентябре 2010 г., в ответ на письмо Ивана Панина, Владимир Воеводский прислал ему свои записи и выразил надежду, что когда-нибудь указанный выше подход будет реализован. В марте 2015 г., после доклада в Институте перспективных исследований, озаглавленного "Framed motives of algebraic varieties (after V. Voevodsky)", ${ }^{4}$ Владимир Воеводский выложил указанные заметки на свою личную страничку. В результате они стали известны широкому кругу математиков, что привлекло к ним огромное внимание специалистов, работающих в данной области. Достаточно отметить, что весь осенний семестр семинара Марка Левина в 2016 г. был посвящён подробнейшему разбору полученных к тому моменту результатов. ${ }^{5}$ Завершение разработки данного аппарата, предсказанного Владимиром Воеводским, принципиально изменит весь ландшафт стабильной мотивной гомотопической топологии и, несомненно, приведёт к новым приложениям.

Вместе с тем, проделав всю фундаментальную работу основателя, Владимир Воеводский переносит свой интерес в другие области математики. Среди прочего он пробует применять мотивные идеи в теории вероятностей, но скоро его полностью поглощает тема оснований математики и компьютерной проверки доказательств. Возможность проверки или хотя бы оценки корректности доказательства с помощью компьютера была давней мечтой математиков (и не только). Современные доказательства становятся всё сложнее и сложнее, и нет уверенности, что авторы и рецензенты проверяют их всегда с необходимой тщательностью.

Но проверять доказательства с помощью компьютера оказалось делом непростым. Главным препятствием была неприспособленность теории множеств - основного языка математики - для этой цели. Правильную замену Владимир Воеводский находит в гомотопической теории типов. Он привносит гомотопические элементы в теорию типов П. Мартин-Лёфа, широко используемую в компьютерных науках. Типы здесь удобно представлять в виде гомотопических типов (что на самом деле является некоторой их реализацией), - тогда различные операции и постулаты теории типов Мартин-Лёфа приобретают ясный топологический смысл. Владимир вводит линейный порядок на типах по их уровню: на нулевом уровне находится одноэлементный тип (аналог стягиваемого пространства в топологии), а дальше индуктивно тип уровня $n$ определяется условием того, что пространство путей (некоторая встроенная операция) между любыми двумя его элементами является типом уровня $n-1$. Тогда типы уровня один можно отождествить с утверждениями, а уровня два - с множествами.

В 2012-2013 г. в Институте перспективных исследований прошёл тематический год по унивалентным основаниям математики, где Владимир был одним из главных организаторов. Результаты этой годовой программы были частично опубликованы в коллективной монографии "Ноmotopy type theory - univalent foundations of mathematics" (Princeton, NJ, 2013).

Владимир Воеводский вводит новую фундаментальную аксиому унивалентности, которая определяет равенство типов и закладывает необходимый фундамент под воз-

\footnotetext{
${ }^{3}$ см. "Framed Correspondences" на странице https://www.math.ias.edu/vladimir/publications.

${ }^{4}$ https://video.ias.edu/tav/2015/0325-IvanPanin; см. также G. Garkusha, I. Panin, Framed motives of algebraic varieties (after V. Voevodsky), arXiv:http://arxiv.org/abs/1409.4372v4.

${ }^{5}$ см. http://www.esaga.uni-due.de/marc.levine/Courses/2016/MotivesSeminarWS/.
} 
водимое им здание. Он также вводит новый метод построения моделей таких теорий и, в частности, строит симплициальную унивалентную модель. Теперь можно было начать переписывать всю математику на новом языке, для чего Владимир Воеводский создаёт библиотеку "Основания" для компьютерного помощника доказательств Coq, куда строго и проверяемо вносит базисные математические понятия. На её основе возникает множество других библиотек, в частности библиотека UniMath. Многие математики привносят свой вклад, и объём формализованной математики быстро растет. Мечтой Владимира было добавить к этой коллекции доказательство гипотезы Блоха-Като, чего, к сожалению, он не успел сделать.

Активная деятельность Владимира Воеводского в этом направлении дала мощный толчок развитию теории типов: за короткий срок в созданной им новой области трудилось уже больше сотни учёных, математиков и специалистов в компьютерных науках. Сам Владимир Воеводский до последних дней работал над унивалентными основаниями математики: за последние пять лет появилось более десяти его работ на эту тему. Он был полон новых идей и планов. Внезапная кончина прервала этот полёт мысли, но его идеи уже проросли и пустили глубокие корни, и область, которую он заложил, уже живёт своей собственной динамичной жизнью, как и созданный им ранее мотивный мир.

В молодости Владимир Воеводский очень любил путешествовать (иногда весьма экзотическими способами) по российским просторам, от ближнего Подмосковья до Дальневосточного побережья, в дальнейшем эта любовь проявлялась в том, что он ежегодно приезжал в Россию, иногда не по одному разу, всегда с большим энтузиазмом выступал на семинарах и конференциях в Москве, а лето любил проводить в дачном домике на реке Хотче. Владимир не был замкнут в рамках одной математики, его интересовал весь спектр культурного, философского и религиозного опыта человечества; долгое время увлекаясь фотографическим искусством, Владимир оставил после себя целую галерею замечательных фотокартин. Он умел дружить, умел заботиться о тех, кто нуждался в помощи. Его близким и друзьям будет очень не хватать его улыбки, его заливистого смеха, разговоров на самые неожиданные темы, а всему математическому миру - его взгляда и руки в тех областях математики, где они были бы завтра очень полезны.

А.А. Бейлинсон, А.С. Вишик, Д. А. Каждан, М. М. Капранов, А.С. Меркуръев, Д.О.Орлов, И. А. Панин, А.А. Суслин, Н.А. Тюрин, Г. Б. Шабат

\section{Список публикаций В. А. Воеводского}

[1] "Правильные триангуляции римановых поверхностей и кривые над полями алгебраических чисел", Докл. АН СССР, 304:2 (1989), 265-268 (совм. с Г. Б. Шабатом); англ. пер.: "Equilateral triangulations of Riemann surfaces, and curves over algebraic number fields", Soviet Math. Dokl., 39:1 (1989), 38-41 (with G. B. Shabat).

[2] "Drawing curves over number fields", The Grothendieck Festschrift, v. III, Progr. Math., 88, Birkhäuser Boston, Boston, MA, 1990, 199-227 (with G. B. Shabat).

[3] “Этальные топологии схем над полями конечного типа над Q", Изв. АН CССР. Сер. матем., 54:6 (1990), 1155-1167; англ. пер.: "Étale topologies of schemes over fields of finite type over Q", Math. USSR-Izv., 37:3 (1991), 511-523.

[4] " $\infty$-группоиды как модель для гомотопической категории", УMH, 45:5(275) (1990), 183-184 (совм. с М. М. Капрановым); англ. пер.: " $\infty$-groupoids as a model for a homotopy category", Russian Math. Surveys, 45:5 (1990), 239-240 (with M. M. Kapranov).

[5] "Свободная $n$-категория, порожденная кубом, ориентированные матроиды и высшие порядки Брюа", Функи. анализ и его прил., 25:1 (1991), 62-65 (совм. 
c M. М. Капрановым); англ. пер.: "Free $n$-category generated by a cube, oriented matroids, and higher Bruhat orders", Funct. Anal. Appl., 25:1 (1991), 50-52 (with M. M. Kapranov).

[6] "Combinatorial-geometric aspects of polycategory theory: pasting schemes and higher Bruhat orders (list of results)", International category theory meeting (Bangor, 1989 and Cambridge, 1990), Cahiers Topologie Géom. Différentielle Catég., 32:1 (1991), 11-27 (with M. M. Kapranov).

[7] " $\infty$-groupoids and homotopy types", International category theory meeting (Bangor, 1989 and Cambridge, 1990), Cahiers Topologie Géom. Différentielle Catég., 32:1 (1991), 29-46 (with M. M. Kapranov).

[8] "О группах Галуа функциональных полей над полями конечного типа над $\mathbb{Q}$ ", УМH, 46:5(281) (1991), 163-164; англ. пер.: "On Galois groups of function fields over fields of finite type over $\mathbb{Q}$ ", Russian Math. Surveys, 46:5 (1991), 202-203.

[9] "Представления Галуа, связанные с гиперболическими кривыми", Изв. АН СССР. Сер. матем., 55:6 (1991), 1331-1342; англ. пер.: "Galois representations connected with hyperbolic curves", Math. USSR-Izv., 39:3 (1992), 1281-1291.

[10] "2-categories and Zamolodchikov tetrahedra equations", Algebraic groups and their generalizations: quantum infinite-dimensional methods (University Park, PA, 1991), Proc. Sympos. Pure Math., 56, Part 2, Amer. Math. Soc., Providence, RI, 1994, 177-259 (with M. M. Kapranov).

[11] "Braided monoidal 2-categories and Manin-Schechtman higher braid groups", J. Pure Appl. Algebra, 92:3 (1994), 241-267 (with M. Kapranov).

[12] Homology of schemes and covariant motives, Ph.D. Thesis, Harvard Univ., Cambridge, MA, 1992, 64 pp.

[13] "A nilpotence theorem for cycles algebraically equivalent to zero", Int. Math. Res. Not., 1995, № 4, 187-198.

[14] "Homology of schemes", Selecta Math. (N. S.), 2:1 (1996), 111-153.

[15] "Singular homology of abstract algebraic varieties", Invent. Math., 123:1 (1996), 61-94 (with A. Suslin).

[16] " $\mathbf{A}^{1}$-homotopy theory", Proceedings of the International Congress of Mathematicians, Vol. I (Berlin, 1998), Doc. Math., 1998, Extra Vol. I, 579-604.

[17] "A ${ }^{1}$-homotopy theory of schemes", Inst. Hautes Études Sci. Publ. Math., 1999, № 90, 45-143 (with F. Morel).

[18] "Voevodsky's Seattle lectures: $K$-theory and motivic cohomology", Notes by C. Weibel, Algebraic K-theory (Seattle, WA, 1997), Proc. Sympos. Pure Math., 67, Amer. Math. Soc., Providence, RI, 1999, 283-303.

[19] "Introduction", Cycles, transfers, and motivic homology theories, Ann. of Math. Stud., 143, Princeton Univ. Press, Princeton, NJ, 2000, 3-9 (with E. M. Friedlander, A. Suslin).

[20] "Relative cycles and Chow sheaves", Cycles, transfers, and motivic homology theories, Ann. of Math. Stud., 143, Princeton Univ. Press, Princeton, NJ, 2000, 10-86 (with A. Suslin).

[21] "Cohomological theory of presheaves with transfers", Cycles, transfers, and motivic homology theories, Ann. of Math. Stud., 143, Princeton Univ. Press, Princeton, NJ, 2000, 87-137.

[22] "Bivariant cycle cohomology", Cycles, transfers, and motivic homology theories, Ann. of Math. Stud., 143, Princeton Univ. Press, Princeton, NJ, 2000, 138-187 (with E. M. Friedlander).

[23] "Triangulated categories of motives over a field", Cycles, transfers, and motivic homology theories, Ann. of Math. Stud., 143, Princeton Univ. Press, Princeton, NJ, 2000, 188-238. 
[24] Cycles, transfers, and motivic homology theories, Ann. of Math. Stud., 143, Princeton Univ. Press, Princeton, NJ, 2000, vi+254 pp. (with E. M. Friedlander, A. Suslin).

[25] "Bloch-Kato conjecture and motivic cohomology with finite coefficients", The arithmetic and geometry of algebraic cycles (Banff, AB, 1998), NATO Sci. Ser. C Math. Phys. Sci., 548, Kluwer Acad. Publ., Dordrecht, 2000, 117-189 (with A. Suslin).

[26] "Open problems in the motivic stable homotopy theory. I", Motives, polylogarithms and Hodge theory, Part I (Irvine, CA, 1998), Int. Press Lect. Ser., 3, 1, Int. Press, Somerville, MA, 2002, 3-34.

[27] "A possible new approach to the motivic spectral sequence for algebraic $K$-theory", Recent progress in homotopy theory (Baltimore, MD, 2000), Contemp. Math., 293, Amer. Math. Soc., Providence, RI, 2002, 371-379.

[28] "Motivic cohomology groups are isomorphic to higher Chow groups in any characteristic", Int. Math. Res. Not., 2002, № 7, 351-355.

[29] "Reduced power operations in motivic cohomology", Publ. Math. Inst. Hautes Études Sci., 2003, № 98, 1-57.

[30] "Motivic cohomology with $\mathbb{Z} / 2$-coefficients", Publ. Math. Inst. Hautes Études Sci., 2003, № 98, 59-104.

[31] "On the zero slice of the sphere spectrum", Алгебраическал геометрия: Методы, связи и приложения, Сборник статей. Посвящается памяти члена-корреспондента РАН Андрея Николаевича Тюрина, Тр. МИАН, 246, Наука, МАИК "Наука/Интерпериодика", М., 2004, 106-115; Proc. Steklov Inst. Math., 246 (2004), 93-102.

[32] Lecture notes on motivic cohomology, Clay Math. Monogr., 2, Amer. Math. Soc., Providence, RI; Clay Math. Inst., Cambridge, MA, 2006, xiv+216 pp. (with C. Mazza, Ch. Weibel).

[33] "An exact sequence for $K_{*}^{M} / 2$ with applications to quadratic forms", Ann. of Math. (2), 165:1 (2007), 1-13 (with D. Orlov, A. Vishik).

[34] "Voevodsky's Nordfjordeid lectures: motivic homotopy theory", Motivic homotopy theory, Universitext, Springer, Berlin, 2007, 147-221 (with O. Röndigs, P. A. Østvær).

[35] Motivic homotopy theory, Lectures from the summer school (Nordfjordeid, 2002), Universitext, Springer-Verlag, Berlin, 2007, x+221 pp. (with B. I. Dundas, M. Levine, P. A. Østvær, O. Röndigs).

[36] "Cancellation theorem", Doc. Math., 2010, Extra vol.: A. A. Suslin sixtieth birthday, 671-685.

[37] "Motives over simplicial schemes", J. K-Theory, 5:1 (2010), 1-38.

[38] "Simplicial radditive functors", J. K-Theory, 5:2 (2010), 201-244.

[39] "Motivic Eilenberg-MacLane spaces", Publ. Math. Inst. Hautes Études Sci., 2010, № 112, 1-99.

[40] "Homotopy theory of simplicial sheaves in completely decomposable topologies", J. Pure Appl. Algebra, 214:8 (2010), 1384-1398.

[41] "Unstable motivic homotopy categories in Nisnevich and cdh-topologies", J. Pure Appl. Algebra, 214:8 (2010), 1399-1406.

[42] "On motivic cohomology with $\mathbb{Z} /$ l-coefficients", Ann. of Math. (2), 174:1 (2011), 401-438.

[43] "Mini-workshop: The homotopy interpretation of constructive type theory" (February 27 - March 05, 2011), Oberwolfach Rep., 8:1 (2011), 609-638 (ed. by V. Voevodsky with S. Awodey, R. Garner, P. Martin-Löf).

[44] "Univalent foundations of mathematics", Logic, language, information and computation (Philadelphia, PA, 2011), Lecture Notes in Comput. Sci., 6642, Lecture Notes in Artificial Intelligence, Springer, Heidelberg, 2011, 4. 
[45] "A univalent formalization of the p-adic numbers", Math. Structures Comput. Sci., 25:5 (2015), 1147-1171 (with Á. Pelayo, M. A. Warren).

[46] "An experimental library of formalized mathematics based on the univalent foundations", Math. Structures Comput. Sci., 25:5 (2015), 1278-1294.

[47] "A C-system defined by a universe category", Theory Appl. Categ., 30 (2015), № 37, $1181-1215$.

[48] "Subsystems and regular quotients of C-systems", A panorama of mathematics: pure and applied, Contemp. Math., 658, Amer. Math. Soc., Providence, RI, 2016, 127-137.

[49] "Products of families of types and $(\Pi, \lambda)$-structures on C-systems", Theory Appl. Categ., 31 (2016), № 36, 1044-1094.

[50] "C-systems defined by universe categories: presheaves", Theory Appl. Categ., 32 (2017), № 3, 53-112.

[51] "The $(\Pi, \lambda)$-structures on the C-systems defined by universe categories", Theory Appl. Categ., 32 (2017), № 4, 113-121.

[52] "Categorical structures for type theory in univalent foundations", Computer science logic 2017, LIPIcs. Leibniz Int. Proc. Inform., 82, Schloss Dagstuhl. Leibniz-Zent. Inform., Wadern, 2017, 16 pp. (with B. Ahrens, P. L. Lumsdaine). 